\title{
Robust Finite-Time Tracking Control Design with Application to Clean Energy Systems
}

\author{
En-Chih Chang ${ }^{a^{*}}$, Yow-Chyi Liu ${ }^{\mathrm{b}}$ \\ ${ }^{a}$ Department of Electrical Engineering, I-Shou University, No.1, Sec. 1, Syuecheng Rd., Dashu District, Kaohsiung City 84001, \\ Taiwan, R.O.C. \\ ${ }^{b}$ Department of Electrical Engineering, Kao Yuan University, No.1821, Jhongshan Rd., Lujhu District, Kaohsiung City 82151, \\ Taiwan, R.O.C.
}

\begin{abstract}
In this paper, a robust finite-time tracking control design for clean energy systems has been developed to maintain a low-total-harmonic-distortion output voltage and a fast dynamic response under various loads. The proposed control combines the properties of a fast-converging sliding mode control (FCSMC) and glowworm swarm optimization (GSO). The FCSMC has a finite system state convergence time unlike the infinite-time exponential convergence of classic sliding mode control, but chattering still occurs under a highly uncertain disturbance. Chattering causes high voltage harmonic distortion in a clean energy system's output. To effectively remove the chattering, the control gains of the FCSMC can be optimally tuned using the GSO. The experimental results obtained from a clean energy system controlled by a digital-signal-processing-based algorithm confirm that the proposed controller can provide a highquality AC output voltage. This paper will be of interest to designers of related clean energy applications because the proposed controller is easier to implement than prior technologies and has a higher tracking precision and quicker convergence speed.
\end{abstract}

Keywords: Fast-converging sliding mode control, glowworm swarm optimization, finite system state convergence time, chattering, clean energy.

\section{Introduction}

Clean energy has gained increasing attention and has broadly been seen in photovoltaic power generation, wind power generation, hydroelectric power generation, etc. On the basis of a previous study [1], a high-performance clean energy system depends upon its static inverter-filter arrangement, which is used to convert DC voltage to a high-quality AC output voltage with low total harmonic distortion (THD) and fast dynamic response. To achieve the abovementioned requirements, a proportional integral (PI) controller is usually used. However, fast transience [2] and a stable output voltage [3] cannot be obtained under uncertain disturbances. Several control solutions derived for clean energy systems have been reported, such as the wavelet transform technique [4], deadbeat control [5], and repetitive control [6], but they are difficult to realize and have complicated algorithms. Sliding mode control (SMC) [7] is a robust trajectory-tracking method owing to its insensitivity to internal parameter variations and external disturbances. The SMC of clean energy systems has been developed [8]; however, its asymptotic stability cannot be ensured via a linear sliding surface. Recently, a fast-converging sliding mode control (FCSMC), which employs a nonlinear sliding surface, has been developed in lieu of a linear sliding surface [9]. By using the nonlinear sliding surface, the FCSMC ensures a finite system state convergence time, but the chattering is a significant shortcoming once a severe uncertain disturbance is applied [10]. Chattering incites unmodeled high-frequency plant dynamics and occasionally causes the system instability. The

\footnotetext{
* Manuscript received July 27, 2017; revised August 28, 2017.

Corresponding author. Tel.: +886-7-6577711 Ext.6642; E-mail address: enchihchang@isu.edu.tw

doi: $10.12720 /$ sgce.7.1.48-52
} 
GSO is a bionic swarm intelligence algorithm [11], which originates from imitating the environment of a glowworm swarm foraging and seeking spouses [12] and has been successfully applied in the power engineering field [13]. Thus, a GSO can be employed to eliminate chattering, which exists in the sliding surface of the FCSMC. By combining FCSMC with GSO, the proposed controller yields a digital signal processing (DSP)-based closed-loop clean energy system with low THD and fast transient under different types of loads. Experiments were used to validate the performance of the proposed controller.

\section{System Modeling}

Figure 1 shows the circuit diagram of a common single-phase inverter in a clean energy system comprising an inductor-capacitor filter and switching component with MOSFET transistors. The LC filter and connected load can be regarded as the plant of the DSP-based feedback system to be controlled.

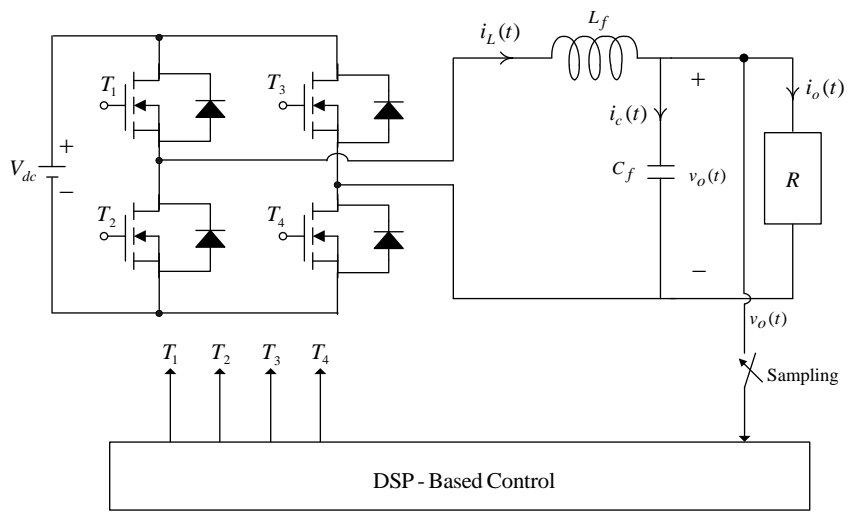

Fig. 1. The single-phase inverter of a clean energy system.

If the output voltage $v_{o}$ tracks a reference voltage $v_{r}$ strictly, the error between $v_{o}$ and $v_{r}$ becomes zero. Therefore, we can define $x_{e 1}=v_{o}-v_{r}$ and $x_{e 2}=\dot{x}_{e 1}$. The error dynamic state space equation can then be derived as follows:

$$
\left[\begin{array}{l}
\dot{x}_{e 1} \\
\dot{x}_{e 2}
\end{array}\right]=\left[\begin{array}{cc}
0 & 1 \\
-a_{1} & -a_{2}
\end{array}\right]\left[\begin{array}{l}
x_{e 1} \\
x_{e 2}
\end{array}\right]+\left[\begin{array}{l}
0 \\
b
\end{array}\right] u+\left[\begin{array}{l}
0 \\
f
\end{array}\right]
$$

where $a_{1}=\frac{1}{L_{f} C_{f}}, \quad a_{2}=\frac{1}{R C_{f}}, \quad b=\frac{K_{p w m}}{L_{f} C_{f}}, \quad K_{p w m}$ is the inverter equivalent gain, and $f=-a_{1} v_{r}-a_{2} \dot{v}_{r}-\ddot{v}_{r}$. The control signal $u$ in Eq. (1) must be designed to suppress the error to zero within a finite time; thus, the control design is described in the following section.

\section{Control Design}

A fast-converging sliding function was constructed as follows:

$$
s(t)=x_{e 1}(t)+\frac{1}{\gamma} x_{e 2}^{\frac{p_{1}}{q_{1}}}(t)
$$

where $p_{1}>q_{1}, p_{1}$ and $q_{1}$ are positive odd numbers $\left(1<\frac{p_{1}}{q_{1}}<2\right)$, and a sliding mode reaching term $\dot{s}=-k|s|^{\beta} \operatorname{sgn}(s)$ was constructed. Then, the control law $u$ can be expressed as follows: 


$$
u(t)=\underbrace{b^{-1}\left[a_{1} x_{e 1}+a_{2} x_{e 2}-\gamma \cdot \frac{q_{1}}{p_{1}} x_{e 2}^{2-\frac{p_{1}}{q_{1}}}\right]}_{u_{e}(t)} \underbrace{-b^{-1}\left[k|s|^{\beta} \operatorname{sgn}(s)\right]}_{u_{s}(t)}, k>0,1>\beta>0,
$$

where $u_{e}$ denotes the equivalent control with nonsingularity and $u_{s}$ displays the sliding control used to compensate for the perturbation. Thus, the system will be driven to the sliding mode $s=0$ and converged within a finite time.

However, FCSMC involves chattering in the clean energy system design due to the changeable load; hence, once the loading becomes a severely uncertain circumstance, the system in Eq. (1) will not provide accurate tracking performance. The control gains in Eq. (3) can be optimally tuned via the GSO, thereby eliminating the chattering. The concept of the GSO is inspired by glowworms that vary the intensity of the luciferin emission such that they glow at various levels. A larger luciferin value indicates higher fitness value and a preferable present location. Within the domain, a glowworm is tempted by the most brilliant neighbor. Also, the position of the glowworms and the luciferin value will be changed after each iteration. The process of the GSO algorithm is represented as follows:

Step 1: The parameters are initialized.

Step 2: For the objective function's search space, the population of the glowworms is placed randomly.

Step 3: The position of the glowworm $i$ is $x_{i}(t)$ and the $J\left(x_{i}(t)\right)$ stands for the objective function value. The luciferin level is, therefore, expressed as follows:

$$
L_{i}(t)=(1-\xi) L_{i}(t-1)+\delta J\left(x_{i}(t)\right)
$$

where $\xi$ denotes the luciferin decay constant $(0<\xi<1)$ and $\delta$ signifies the luciferin enhancement constant.

Step 4: Within a variable neighborhood range $r_{d_{i}}(t)\left(0<r_{d_{i}}<r_{s}\right)$, each glowworm decides its neighbor and a larger luciferin value is obtained. Thus, each glowworm $i$ will move toward a neighbor $j$ with a certain probability as follows:

$$
p_{i j}(t)=\frac{L_{j}(t)-L_{i}(t)}{\sum_{n \in N_{i}} L_{n}(t)-L_{i}(t)}
$$

where $N_{i}$ is the set of neighbors of glowworm $i$.

Step 5: The position of glowworm $i$ can be determined using Eq. (6):

$$
x_{i}(t+1)=x_{i}(t)+s_{z} \cdot\left(\frac{x_{j}(t)-x_{i}(t)}{\left\|x_{j}(t)-x_{i}(t)\right\|}\right)
$$

where $s_{z}$ is the step size.

Step 6: The neighborhood range can be determined using Eq. (7):

$$
r_{d_{i}}(t+1)=\min \left\{r_{s}, \max \left\{0, r_{d_{i}}(t)+\psi\left(n_{t}-\left|N_{i}(t)\right|\right)\right\}\right\}
$$

where $\psi$ is a constant and $n_{t}$ governs the number of neighbors.

\section{Experimental Results}

The parameters of the clean energy system are listed as follows: DC-bus voltage $V_{d c}=210 \mathrm{~V}$; output voltage $v_{o}=110 \mathrm{~V}$; output frequency $f=60 \mathrm{~Hz}$; filter inductor $L=0.1 \mathrm{mH}$; filter capacitor $C=5 \mu \mathrm{F}$; switching frequency $f_{s w}=20 \mathrm{kHz}$; and rated loading $R=12 \Omega$. Figure 2 shows the waveforms with the proposed controller under TRIAC loading. The transient response of the proposed controller is very fast 
at the firing angle and the voltage variation is small. As shown in Fig. 3, the waveforms with the classic SMC under TRIAC loading display a large voltage variation and a slow voltage recovery time. The experimental results obtained for the clean energy system under rectifier load, comprising a full wave rectifier with a parallel resistor $(50 \Omega)$ and capacitor filter $(200 \mu \mathrm{F})$ using the proposed controller and the classic SMC are shown in Figs. 4 and 5, respectively. The figures show that the output voltage of the proposed controlled clean energy system has lower distortion than that of the classic sliding mode controlled clean energy system. The proposed controller is capable of yielding a good steady state and transient state, and it shows a significant improvement in reducing the THD of the output voltage under rectifier-type loading conditions. The output voltage exhibits a low \%THD of $1.02 \%$, with the proposed controller. For comparison, the output voltage obtained using the classic SMC has a high \%THD of $7.45 \%$.

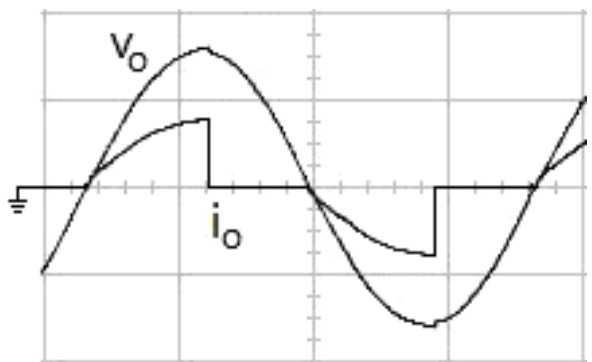

Fig. 2. The proposed controller under TRIAC loading (vert. : $100 \mathrm{~V} /$ div; vert. : $20 \mathrm{~A} /$ div; hor. : 5 ms/div).

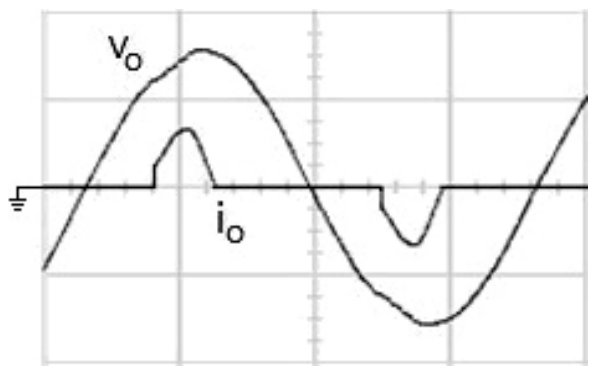

Fig. 4. The proposed controller under rectifier loading (vert. : $100 \mathrm{~V} /$ div; vert. : $25 \mathrm{~A} /$ div; hor. : 5 $\mathrm{ms} / \mathrm{div})$.

\section{Conclusions}

In this paper, we developed a GSO-based FCSMC to reject uncertain system disturbances. The FCSMC forces tracking errors to zero within a finite time, and the GSO helps eliminate chattering. The experimental results show that a low THD, fast dynamics, and chattering alleviation are achieved under varied loads.

\section{Acknowledgements}

This work was supported by the Ministry of Science and Technology of Taiwan, R.O.C., under contract number MOST106-2221-E-214-031.

\section{References}

[1] Branko LD, Branko B. Power Electronics: Converters and Regulators. New York: Springer; 2015. 
[2] Shin HB, Park JG. Anti-windup PID controller with integral state predictor for variable-speed motor drives. IEEE Trans. Ind. Electron., 2012; 59(3):1509-1516.

[3] Rebeiro RS, Uddin MN. Performance analysis of an FLC-based online adaptation of both hysteresis and PI controllers for IPMSM drive. IEEE Trans. on Industry Applications, 2012; 48(1):12-19.

[4] Saleh SA, Rahman MA. Experimental performances of the single-phase wavelet-modulated inverter. IEEE Trans. on Power Electronics, 2011; 26(9):2650-2661.

[5] Zhang XG, Zhang WJ, Chen JM, Xu DG. Deadbeat control strategy of circulating currents in parallel connection system of three-phase PWM converter. IEEE Trans. on Energy Conversion, 2014; 29(2):406-417.

[6] Chen D, Zhang JM, Qian ZM. An improved repetitive control scheme for grid-connected inverter with frequency-adaptive capability. IEEE Trans. on Industrial Electronics, 2013; 60(2):814-823.

[7] Azar AT, Zhu QM. Advances and applications in sliding mode control systems. New York: Springer; 2015.

[8] Tan SC, Lai YM, Tse CK. Sliding mode control of switching power converters: techniques and implementation. CRC Press; 2012.

[9] Mohammadpour A, Ajami A, Zadeh SG, Aghababa MP, Mahboubi BM. Robust terminal sliding mode power flow controller using unified power flow controller with adaptive observer and local measurement. IET Generation, Transmission \& Distribution, 2014; 8(10):1712- 1723.

[10] Galias Z, Yu XH. Dynamical behaviors of discretized second-order terminal sliding-mode control systems. IEEE Trans. on Circuits and Systems II: Express Briefs, 2012; 59(9):597-601.

[11] Yu Z, Yang XM. Full glowworm swarm optimization algorithm for whole-set orders scheduling in single machine. Scientific World Journal, 2013:1-6.

[12] Li MG, Wang X, Gong Y, Liu YY, Jiang CW. Binary glowworm swarm optimization for unit commitment. Journal of Modern Power Systems and Clean Energy, 2014; 2(4):357-365.

[13] Lenin K, Reddy BR. Suryakalavathi M. Reduction of real power loss by using double glow-worms swarm co-evolution optimization algorithm based Levy flights. International Journal of Novel Research in Electrical and Mechanical Engineering, 2014; $1(1): 1-12$. 